\title{
Focus on optimization of early antimicrobial therapy in ICU-acquired infections
}

\author{
Jean-François Timsit ${ }^{12^{*}}$, José-Artur Paiva ${ }^{3,4}$ and Matteo Bassetti ${ }^{5,6}$
}

๑ 2016 Springer-Verlag Berlin Heidelberg and ESICM

\section{Introduction}

The purpose of a new empirical antimicrobial therapy is to deliver adequate treatment early while limiting antibiotic selection pressure and antibiotic resistance. The choice of molecules will be guided by the guidelines and the knowledge of previous multiresistant bacteria (MRB) carriers, secondarily adapted according to microbiological data. Finally, early optimal pharmacokinetics/pharmacodynamics (PK/PD) of empirical therapy is a necessary and safe starting point from where de-escalation can take place. The antimicrobial chosen for de-escalation should be used according to antibiotic susceptibility testing, and using proper doses to ensure appropriate PK during targeted therapy.

\section{Early recognition of multiresistant-bacteria carriers} Healthcare facilities, particularly acute care and ICU, are important sites for the development of antimicrobial resistance. The intensity of antimicrobial agents use, together with populations highly susceptible to infection, creates an environment that facilitates both the emergence and the transmission of MRB. Readmission of patients colonized with MRB is an important factor in the nosocomial dynamics of MRB. It creates a "feedback loop" with reintroduction of pathogens into the ward that can colonize or infect new patients. Several studies have assessed the duration of carriage of MRB in varying settings, yielding large differences in colonization duration, from about 1 month to a persistence lasting more than 40 months. Haverkate and coworkers showed that the median times until clearance were 4.8 months for

\footnotetext{
*Correspondence: Jean-francois.timsit@bch.aphp.fr

1 Université Paris Diderot/Hôpital Bichat, Réanimation Médicale et des maladies infectieuses, 46 rue Henri Huchard, 75018 Paris, France

Full author information is available at the end of the article
}

all MRB together, around 1.4 months for Enterobacteriaceae, and 1 month for methicillin-resistant Staphylococcus aureus (MRSA) [1].

Around $20 \%$ of MRSA-infected patients never clear their colonization, and older age has been reported to be associated with longer duration of MRSA colonization [2]. Early caloric negative-energy balance is an independent determinant of MRSA colonization and of late-onset infection in mechanically ventilated patients. Feeding prescriptions aimed at limiting the energy deficit during the first week of ICU stay could contribute to MRSA prevention [3]. Based on a recent systematic review of 16 studies (13 observational studies and three randomized controlled trials), the estimated time-to-clearance after documented colonization was 88 weeks for MRSA-colonized patients, and 26 weeks for vancomycin-resistant enterococci-colonized patients [4]. Duration of colonization with $E$. coli resistant to quinolones was 6 months on average, with variations according to indwelling devices [5]. The duration of colonization should be taken into account for the choice of empirical therapy in case of further infections, and for placing patients under contact precautions until the results of the new surveillance cultures in case of hospital readmission.

\section{Rapid diagnostic tests for selecting adequate antimicrobials in severe infections}

With the rapid increase of bacteria that are resistant to almost all antimicrobials, the early selection of adequate antimicrobial agents becomes difficult in case of sepsis. Ideally, antimicrobial treatment must immediately cover microorganisms responsible for infection and avoid overuse of broad-spectrum antimicrobials.

Classical microbiological tests require at least $24-48 \mathrm{~h}$ for microorganism identification and 48-72 h for antibiotic susceptibility profile determination. Furthermore

\section{实


the early detection of microorganisms may lack sensitivity, especially if patients received previous antimicrobial therapy.

In order to solve this issue, new rapid diagnostic tests have been developed. Many novel rapid nucleic acid amplification or mass spectrometry-based techniques provide rapid identification of targeted microorganisms. Some are also able to detect some resistance genes.

Real-time PCR assay that targets ribosomal sequences of a panel of selected bacteria and fungi has extensively been studied in patients with suspected sepsis. Warhurst et al. provided results of a study on 1006 episodes of suspected bloodstream infections (BSIs) [6]. Of 77 BSIs diagnosed with traditional methods, only 44 had the same pathogens identified with RT PCR. In 133 other cases, only the PCR was positive and may reflect alternately a true BSI missed by blood culture or false positive results. This result was confirmed by a systematic review [7]. From 41 phase III diagnostic accuracy studies, summary sensitivity and specificity for SeptiFast ${ }^{\mathrm{TM}}$ compared with blood cultures were 0.68 (95\% CI 0.63-0.73) and 0.86 (95\% CI 0.84-0.89), respectively.

Nucleic acid amplification testing (NAAT), mass spectrometry, and fluorescence in situ hybridization (FISH) identification techniques can all identify pathogens in blood culture or respiratory secretion within $6 \mathrm{~h}$, and NAAT and mass spectrometry can identify selected antibiotic resistance genes (vancomycin: $\operatorname{Van} A / \operatorname{Van} B$, methicillin: $M e c A$, and carbapenem: carbapenemase-resistant enterobacteriaceae). A study involving 616 BSIs and 185 pneumonia cases tested the PlexID ${ }^{\mathrm{TM}}$ platform, which is able to detect 800 relevant pathogens. The system identified 55 of the 68 positive blood cultures, but was also positive in 173/625 of negative blood cultures [8]. The interpretation and therapeutic implications of recovering positive $\mathrm{PCR}$ in the absence of positive microbial culture remain debated.

FISH-based microscopy identification and antibiotic susceptibility test (ID/AST) systems can evaluate antibiotic susceptibility from blood cultures or respiratory secretion on a previously defined panel of phenotypic growth pattern analysis. One recent pilot study reported data from a new accelerate ID/AST automated microscopy system was able to detect MRB in bronchoalveolar lavage after $5 \pm 7 \mathrm{~h}$ of culture and $5 \mathrm{~h}$ of analysis with a $100 \%$ sensitivity and a $97 \%$ specificity [9].

These new techniques only detect the presence of genes of the pathogen and of resistance but are neither able to differentiate alive and dead pathogens nor give information regarding phenotypic antimicrobial susceptibility. The pattern of resistance is not able to detect genes coding for extended broad-spectrum beta-lactamases. Further studies with a better selection of pathogens more adapted to the clinical situation and a quantification of the detected DNA are warranted.

In the meantime, one should keep in mind that rapid antimicrobial susceptibility testing combined with mass spectrometry could give identification and accurate antibiotic susceptibility results within 1 day of sampling [10]. Rapid detection of enzymes such as extended-spectrum beta-lactamases, $A m p C$, and carbapenemases is also possible within a few hours using biochemical techniques (ca. $90 \%$ sensitivity and ca. $90 \%$ specificity) [11].

\section{A plea for PK-oriented de-escalation through antimicrobial stewardship}

Compared to other patients, critically ill patients exhibit more unpredictable antibiotic PK, more severe infections, more resistant microorganisms, and more probable collateral damage. PK variability, mainly marked for hydrophilic antibiotics like beta-lactams, glycopeptides, and aminoglycosides, is caused by widely variable drug clearance (due to augmented renal clearance, acute kidney injury, renal replacement therapy, or extracorporeal membrane oxygenation) and also by variations in volume of distribution and protein binding [12].

The reported lack of effect of combination therapy for severe gram-negative infections and the decreased susceptibility of microorganisms may suggest that higher aminoglycoside doses should be used for initial empiric therapy (amikacin 25-30 $\mathrm{mg} / \mathrm{kg}$, gentamicin and tobramycin 7-9 $\mathrm{mg} / \mathrm{kg}$ ) and that the Cmax of the previous dose (30 min after administration) and the pathogen minimum inhibitory concentration (MIC) should be measured to optimize therapy, in order to target $\mathrm{Cmax} /$ MIC ratios of 8-10 for directed therapy [13].

Determining the steady-state vancomycin concentration in case of continuous infusion after a loading dose, or the residual concentration in the case of discontinuous administrations, is worth the effort. In MRSA with vancomycin MIC $>1 \mathrm{mg} / \mathrm{l}$, the vancomycin dose which is needed to achieve an AUC/MIC $>400$, a ratio associated with a favorable outcome, is in the range associated with nephrotoxicity. Daptomycin, proposed as an alternative, shows marked PK variability due to high clearance; in critically ill patients, doses of $8-10 \mathrm{mg} / \mathrm{kg} /$ day are necessary at therapy onset [14].

Regarding beta-lactams, the DALI study showed over 500-fold differences in plasma drug concentrations [15]. As a result of the rising beta-lactam resistance in Enterobacteriaceae and Pseudomonas aeruginosa and the PK specificities in critically ill patients, administering beta-lactam antibiotics by continuous or prolonged infusion to treat severe infections should be considered, both to improve clinical success and avoid resistance development. We should move to measure residual 
plasma concentrations in discontinuous and prolonged administration, or steady-state plasma concentrations in continuous infusion, aiming to maintain the plasma concentrations of beta-lactam antibiotics above the MIC for at least $70 \%$ of the time.

Therefore, hitting hard and fast with an appropriate antibiotic, the core idea of the antibiotic deescalation strategy implies using good PK-oriented prescription practices and monitoring. This modern exercise of de-escalation needs an antimicrobial stewardship programme for a perfect implementation.

\begin{abstract}
Author details
${ }^{1}$ Université Paris Diderot/Hôpital Bichat, Réanimation Médicale et des maladies infectieuses, 46 rue Henri Huchard, 75018 Paris, France. ${ }^{2}$ U1137-IAME Team 5, DeSCID: Decision SCiences in Infectious Diseases, Control and Care, Inserm/Université Paris Diderot, Sorbonne Paris Cité, 75018 Paris, France. ${ }^{3}$ Department of Emergency and Intensive Care, Centro Hospitalar Sao Joao, Porto, Portugal. ${ }^{4}$ Department of Medicine, Faculty of Medicine, University of Porto, Porto, Portugal. ${ }^{5}$ Clinica Malattie Infettive, Azienda Ospedaliera Universitaria Santa Maria della Misericorida, Udine, Italy. ${ }^{6}$ University of Udine School of Medicine, Udine, Italy.
\end{abstract}

Received: 30 June 2016 Accepted: 1 August 2016

Published online: 11 August 2016

\section{References}

1. Haverkate MR, Derde LPG, Brun-Buisson C, Bonten MJM, Bootsma MCJ (2014) Duration of colonization with antimicrobial-resistant bacteria after ICU discharge. Intensive Care Med 40:564-571

2. Cluzet VC, Gerber JS, Nachamkin I, Metlay JP, Zaoutis TE, Davis MF, Julian KG, Linkin DR, Coffin SE, Margolis DJ, Hollander JE, Bilker WB, Han X, Mistry RD, Gavin LJ, Tolomeo P, Wise JA, Wheeler MK, Hu B, Fishman NO, Royer D, Lautenbach E (2015) Risk factors for recurrent colonization with methicillin-resistant Staphylococcus aureus in community-dwelling adults and children. Infect Control Hosp Epidemiol 36:786-793

3. Ekpe K, Novara A, Mainardi JL, Fagon JY, Faisy C (2014) Methicillin-resistant Staphylococcus aureus bloodstream infections are associated with a higher energy deficit than other ICU-acquired bacteremia. Intensive Care Med 40:1878-1887

4. Shenoy ES, Paras ML, Noubary F, Walensky RP, Hooper DC (2014) Natural history of colonization with methicillin-resistant Staphylococcus aureus (MRSA) and vancomycin-resistant Enterococcus (VRE): a systematic review. BMC Infect Dis 14:177
5. Ismail MD, Luo T, McNamara S, Lansing B, Koo E, Mody L, Foxman B (2016) Long-term carriage of ciprofloxacin-resistant Escherichia coli isolates in high-risk nursing home residents. Infect Control Hosp Epidemiol 37:440-447

6. Warhurst G, Maddi S, Dunn G, Ghrew M, Chadwick P, Alexander P, Bentley A, Moore J, Sharman M, Carlson GL, Young D, Dark P (2015) Diagnostic accuracy of SeptiFast multi-pathogen real-time PCR in the setting of suspected healthcare-associated bloodstream infection. Intensive Care Med 41:86-93

7. Dark P, Blackwood B, Gates S, McAuley D, Perkins GD, McMullan R, Wilson C, Graham D, Timms K, Warhurst G (2015) Accuracy of LightCycler ${ }^{\circledR}$ SeptiFast for the detection and identification of pathogens in the blood of patients with suspected sepsis: a systematic review and meta-analysis. Intensive Care Med 41:21-33

8. Vincent JL, Brealey D, Libert N, Abidi NE, O'Dwyer M, Zacharowski K, Mikaszewska-Sokolewicz M, Schrenzel J, Simon F, Wilks M, Picard-Maureau $M$, Chalfin DB, Ecker DJ, Sampath $R$, Singer M (2015) Rapid diagnosis of infection in the critically ill, a multicenter study of molecular detection in bloodstream infections, pneumonia, and sterile site infections. Crit Care Med 43:2283-2291

9. Douglas IS, Price CS, Overdier KH, Wolken RF, Metzger SW, Hance KR, Howson DC (2015) Rapid automated microscopy for microbiological surveillance of ventilator-associated pneumonia. Am J Respir Crit Care Med 191:566-573

10. Le Dorze M, Gault N, Foucrier A, Ruppe E, Mourvillier B, Woerther PL, Birgand G, Montravers P, Dilly MP, Tubach F, Andremont A, Timsit JF, Wolff $M$, Armand-Lefevre L (2015) Performance and impact of a rapid method combining mass spectrometry and direct antimicrobial susceptibility testing on treatment adequacy of patients with ventilator-associated pneumonia. Clin Microbiol Infect 21(468):e461-e466

11. Poirel L, Fernandez J, Nordmann P (2016) Comparison of three biochemical tests for rapid detection of extended-spectrum beta-lactamaseproducing Enterobacteriaceae. J Clin Microbiol 54:423-427

12. Roberts JA, Taccone FS, Lipman J (2015) Understanding PK/PD. Intensive Care Med. doi:10.1007/s00134-015-4032-6

13. Matthaiou DK, De Waele J, Dimopoulos G (2014) What is new in the use of aminoglycosides in critically ill patients? Intensive Care Med 40:1553-1555

14. Falcone M, Russo A, Venditti M, Novelli A, Pai MP (2013) Considerations for higher doses of daptomycin in critically ill patients with methicillinresistant Staphylococcus aureus bacteremia. Clin Infect Dis 57:1568-1576

15. Roberts JA, Paul SK, Akova M, Bassetti M, De Waele JJ, Dimopoulos G, Kaukonen KM, Koulenti D, Martin C, Montravers P, Rello J, Rhodes A, Starr T, Wallis SC, Lipman J (2014) DALI: defining antibiotic levels in intensive care unit patients: are current beta-lactam antibiotic doses sufficient for critically ill patients? Clin Infect Dis 58:1072-1083 